\title{
THE NUMBER OF SOLUTIONS OF A TRINOMIAL CONGRUENCE INVOLVING A $k$ TH POWER AND A SQUARE
}

\author{
BY J. T. CROSS
}

Communicated by G. B. Huff, September 28, 1962

Let $K$ denote a finite extension of the rational number field and $D$ the domain of algebraic integers of $K$. Let $P$ be a prime ideal of $D$ having norm $N(P)=p^{h}=q$, where $h$ is a positive integer and $p$ is an odd rational prime number. This announcement is concerned with the number of solutions of the trinomial congruence,

$$
X^{k}+\alpha Y^{2} \equiv \rho\left(\bmod P^{r}\right),
$$

where $\alpha$ and $\rho$ are in $D$ with $\rho$ arbitrary and $(\alpha, P)=1, r$ is a positive integer, $k$ is a positive integer such that $(k, p)=1$, and $d=(k, q-1)$ $>1$. Let $C$ denote an ideal of $D$ such that $(P, C)=1$ and $P C=(\theta)$ is principal, and let $b$ be the greatest integer $n$ such that $0 \leqq n \leqq r$ and $P^{n} \mid \rho$. Then we may put

$$
\rho \equiv \eta \theta^{b}\left(\bmod P^{r}\right), \quad(\eta, P)=1,
$$

where $\eta$ is uniquely determined $\left(\bmod P^{r-b}\right)$ if $b<r$.

In Theorems 1-8 we give formulas for the number $Q_{r}(\rho)$ of solutions of (1). Solvability criteria are obtained as corollaries of these theorems. (We remark that if $\rho \equiv 0\left(\bmod P^{r}\right)$, then $(1)$ has the trivial solution $(0,0)$.) The formulas given in this note follow directly from more general theorems proved for congruences $\left(\bmod P^{r}\right)$ involving a $k$ th power and an arbitrary number of squares [2].

If $r=1$, the congruence (1) amounts to an equation in a Galois field of order $q$. For discussions of general trinomial congruences in a finite field, particular reference is made to Vandiver [7] who has published several pertinent papers in recent years. A number of authors have considered the special case of (1) with $r=1$ and $K$ the rational field; in particular we mention Frattini [3], E. Lehmer [5], and Manin [6]. For a discussion of trinomial congruences in algebraic number fields, see Cohen's paper [1].

We need the following notation:

$$
b=L k+I \quad(0 \leqq I<k) ; \quad \zeta=(-\alpha / P), \quad \tau=(-\eta / P),
$$

where $(\beta / P)$ denotes the Legendre symbol in $D$.

Let $Q(\eta)=Q_{1}(\eta)$ denote the number of solutions of

$$
X^{k}+\alpha Y^{2} \equiv \eta(\bmod P), \quad(\eta, P)=1 .
$$


Theorems 1-4 and 7-8 below contain explicit formulas for the number of solutions of (1), while Theorems 5 and 6 are reduction formulas which give the number of solutions of (1) in terms of the number of solutions of (4). Theorems 5 and 6 apply if $d>2, \rho \neq \equiv 0\left(\bmod P^{r}\right)$, $b \equiv 0(\bmod k)$, and $b k$ is even; under these conditions it is not possible to give explicit formulas for $Q_{r}(\rho)$. Davenport and Hasse [4] have shown that $Q(\eta) \geqq q-(d-1) \sqrt{ } q$, a result which we utilize in Corollary 7.

THEOREM 1. If $k$ is odd and $r>b \not \equiv 0(\bmod k)$, then $Q_{r}(\rho)\left(q^{k-2}-1\right) / q^{r-1}$ $=(q-1)\left(q^{(k / 2-1) L+k-2}-1\right)$ for $L$ even, $I$ odd or for $L$ even, $I$ even, $\tau \zeta=-1 ;(q-1)\left(q^{(k / 2-1) L+k-2}-1\right)+2\left(q^{k-2}-1\right) q^{(k / 2-1) L+I / 2}$ for $L$ even, I even, $\tau \zeta=1 ;(q-1)\left(q^{(k / 2-1)(L+1)}-1\right)$ for $L$ odd, I even, or for $L$ odd, $I$ odd, $\tau \zeta=-1 ;(q-1)\left(q^{(k / 2-1)(L+1)}-1\right)+2\left(q^{k-2}-1\right) q^{(k / 2-1) L+1 / 2}$ for $L$ odd, $I$ odd,$\tau \xi=1$.

Corollary 1. If $k$ is odd and $b \neq 0(\bmod k)$, then (1) is solvable.

ThEOREM 2. If $k=2$ and $r>b \neq 0(\bmod 2)$, then $Q_{r}(\rho) / q^{r-1}=0$ for $\zeta=-1 ; 2(q-1)(L+1)$ for $\zeta=1$. If $k$ is even, $k>2$, and $r>b \neq 0(\bmod k)$, then $Q_{r}(\rho)\left(q^{k / 2-1}-1\right) / q^{r-1}=0$ for $I$ odd,$\zeta=-1$, or for $I$ even, $\zeta=-1$ $=-\tau ; 2(q-1)\left(q^{(k / 2-1)(L+1)}-1\right)$ for $I$ odd,$\zeta=1$, or for $I$ even, $\zeta=1=-\tau$; $2 q^{(k / 2-1) L+I / 2}\left(q^{k / 2-1}-1\right)$ for $I$ even, $\zeta=-1=\tau ; 2(q-1)\left(q^{(k / 2-1)(L+1)}-1\right)$ $+2 q^{(k / 2-1) L+I / 2}\left(q^{k / 2-1}-1\right)$ for $I$ even, $\zeta=1=\tau$.

CoRollary 2. If $k$ is even and $r>b \neq 0(\bmod k)$, then (a) If $I$ is odd, the congruence (1) is insolvable $\rightleftarrows \zeta=-1$.

(b) If $I$ is even, the congruence (1) is insolvable $\rightleftarrows \zeta=-1=-\tau$.

Theorem 3. If $k=2$ and $r>b \equiv 0(\bmod 2)$, then $Q_{r}(\rho) / q^{r-1}$ $=(q-1)(1+2 L)$ for $\zeta=1 ; q+1$ for $\zeta=-1$. If $d=2<k$ and $r>b \equiv 0(\bmod k)$, then

$$
Q_{r}(\rho)\left(q^{k / 2-1}-1\right) / q^{r-1}=(q-1)\left(q^{(k / 2-1)(L+1)}+q^{(k / 2-1) L}-2\right)
$$

for $\zeta=; 1(q+1) q^{(k / 2-1) L}\left(q^{k / 2-1}-1\right)$ for $\zeta=-1$.

Corollary 3. If $d=2$ and $b \equiv 0(\bmod k)$, then (1) is solvable.

THEOREM 4. If $k$ is odd, $b$ is odd and $r>b \equiv 0(\bmod k)$, then $Q_{r}(\rho)\left(q^{k-2}-1\right) / q^{r-1}=(q-1)\left(q^{(k / 2-1)(L+1)}-1\right)$ for $\eta$ not a kth power $(\bmod P) ;(q-1)\left(q^{(k / 2-1)(L+1)}-1\right)+d q^{(k / 2-1) L+1 / 2}\left(q^{k-2}-1\right)$ for $\eta a k t h$ power $(\bmod P)$.

Corollary 4. If $k$ is odd, $b$ is odd, and $b \equiv 0(\bmod k)$, then (1) is solvable. 
TheOREM 5. If $k$ is even, $d>2$, and $r>b \equiv 0(\bmod k)$, then $Q_{r}(\rho) / q^{r-1}$ $=q^{(k / 2-1) L} Q(\eta)$ for $\zeta=-1$ and

$$
\begin{array}{r}
Q_{r}(\rho)\left(q^{k / 2-1}-1\right) / q^{r-1} \\
=q^{(k / 2-1) / L}\left\{q^{k / 2}+q-2+(Q(\eta)-q) \cdot\left(q^{k / 2-1}-1\right)\right\}-2(q-1) \\
\quad \text { for } \zeta=1 .
\end{array}
$$

CoRollary 5. If $k$ is even, $d>2$, and $r>b \equiv 0(\bmod k)$, then

(a) If $\zeta=-1, Q_{r}(\rho)=0 \rightleftarrows Q(\eta)=0$.

(b) If $\zeta=1, Q_{r}(\rho)=0 \rightleftarrows Q(\eta)=0$ and $L=0$.

Theorem 6. If $k$ is odd, $b$ is even and $r>b \equiv 0(\bmod k)$, then $Q_{r}(\rho)\left(q^{k-2}-1\right) / q^{r-1}$

$$
=1-q+q^{(k / 2-1) L}\left\{q^{k-1}-1+(Q(\eta)-q)\left(q^{k-2}-1\right)\right\} .
$$

Corollary 6. If $k$ is odd, $b$ is even, and $r>b \equiv 0(\bmod k)$, then $Q_{r}(\rho)=0 \rightleftarrows Q(\eta)=0$ and $L=0$.

Since $Q(\eta) \geqq q-(d-1) \sqrt{q}$, one obtains from Corollaries 5 and 6 ,

Corollary 7. If $d>2, b k$ is even, and $r>b \equiv 0(\bmod k)$, then (1) is solvable if $q>(d-1)^{2}$; moreover, (1) is solvable for arbitrary $q$ if $L \neq 0$ and $k$ is odd, or if $L \neq 0$ and $\zeta=1$.

For completeness, the following formulas in the case $b=r\left(\rho \equiv 0\left(\bmod P^{r}\right)\right)$ are also included.

THEOREM 7. If $k=2$ and $b=r$, then $Q_{r}(\rho) / q^{r-1}=q+(q-1) r$ for $\zeta=1$; $q$ for $\zeta=-1, r$ even; 1 for $\zeta=-1, r$ odd. If $k$ is even, $k>2$ and $b=r$, then $Q_{r}(\rho) / q^{r-1}=q^{(k / 2-1) L+1}$ for $I=0, \zeta=-1$;

$$
\left\{q^{(k / 2-1) L}\left(q^{k / 2}+q-2\right)-2(q-1)\right\} /\left(q^{k / 2-1}-1\right) \text { for } I=0, \zeta=1 \text {; }
$$

$q^{(k / 2-1) L+I / 2}$ for $I$ even, $I>0, \zeta=-1 ; q^{(k / 2-1) L+(I-1) / 2}$ for $I$ odd,$\zeta=-1$; $\left\{q^{(k / 2-1) L}\left(q^{k / 2}+q-2\right)-2(q-1)\right\} /\left(q^{k / 2-1}-1\right)+q^{(k / 2-1) L}\left(q^{I / 2}+q-2\right)$ for $I$ even, $I>0, \quad \zeta=1 ; \quad\left\{q^{(k / 2-1) L}\left(q^{k / 2}+q-2\right)-2(q-1)\right\} /\left(q^{k / 2-1}-1\right)$ $+q^{(k / 2-1) L}\left(q^{(I-1) / 2}+q-2\right)$ for $I$ odd,$\zeta=1$.

TheOREM 8. If $k$ is odd and $b=r$, then

$$
\begin{aligned}
Q_{r}(\rho) / q^{r-1}= & \left\{q^{(k / 2-1) L}\left(q^{k-1}-1\right)-q+1\right\} /\left(q^{k-2}-1\right) \\
& +q^{(k / 2-1) L}\left(q^{I / 2}-1\right) \text { for L even, } I \text { even } ;
\end{aligned}
$$

$\left\{q^{(k / 2-1) L}\left(q^{k-1}-1\right)-q+1\right\} /\left(q^{k-2}-1\right)+q^{(k / 2-1) L}\left(q^{(1-1) / 2}-1\right)$ for $L$ even, I odd; $\left\{q^{(k / 2-1) L}\left(q^{k-3 / 2}+q^{k / 2}-q^{k / 2-1}-q^{1 / 2}\right)-q+1\right\} /\left(q^{k-2}-1\right)$ for $L$ odd, $I=0$; 


$$
\begin{aligned}
& \left\{q^{(k / 2-1) L}\left(q^{k-8 / 2}+q^{k / 2}-q^{k / 2-1}-q^{1 / 2}\right)-q+1\right\} /\left(q^{k-2}-1\right) \\
& +q^{(k / 2-1) L+1 / 2}\left(q^{I / 2-1}-1\right) \text { for } L \text { odd, } I \text { even, } I>0 \text {; } \\
& \left\{q^{(k / 2-1) L}\left(q^{k-3 / 2}+q^{k / 2}-q^{k / 2-1}-q^{1 / 2}\right)-q+1\right\} /\left(q^{k-2}-1\right) \\
& +q^{(k / 2-1) L+1 / 2}\left(q^{(I-1) / 2}-1\right) \text { for } L \text { odd, I odd. }
\end{aligned}
$$

We now apply the formulas to a few examples, letting $K$ be the rational field. $X^{3}+Y^{2} \equiv 2 \cdot 7^{3}\left(\bmod 7^{4}\right)$ has 2,058 solutions by Theorem $4 ; X^{4}+2 Y^{2} \equiv 25(\bmod 125)$ has no solutions by Corollary 2; $X^{4}+Y^{2} \equiv 3 \cdot 5^{4}\left(\bmod 5^{5}\right)$ has 5,000 solutions by Theorem $5 ; X^{6}+Y^{2}$ $\equiv 6 \cdot 7^{6}\left(\bmod 7^{7}\right)$ has no solutions by Corollary 5 .

\section{BIBLIOGRAPHY}

1. Eckford Cohen, Binary congruences in algebraic number fields, Proc. Nat. Acad. Sci. 42 (1956), 120-122.

2. J. T. Cross, The number of solutions of certain types of congruences in algebraic number fields, (to appear).

3. G. Frattini, Intorno ad un teorema di Lagrange, Atti Reale Accad. Lincei Rend. 1 (1885), 136-142.

4. H. Davenport and H. Hasse, Die Nullstellen der Kongruenz-zetafunktionen in gewissen zyklischen Fällen, J. Reine Angew. Math. 172 (1935), 151-182.

5. Emma Lehmer, On the number of solutions of $u^{k}+D=w^{2}(\bmod p)$, Pacific J. Math. 5 (1955), 103-118.

6. Yu. I. Manin, On cubic congruences to a prime modulus, Amer. Math. Soc. Transl. (2) 13 (1960), 1-7.

7. H. S. Vandiver, On the number of solutions of certain non-homogeneous trinomial equations in a finite field, Proc. Nat. Acad. Sci. 31 (1945), 170-175.

The University of TenNessee AND

The UNIVERSITY OF THE SOUTH 